\title{
Tazobactam Sodium
}

National Cancer Institute

\section{Source}

National Cancer Institute. Tazobactam Sodium. NCI Thesaurus. Code C1242.

The sodium salt form of tazobactam, a penicillanic acid sulfone derivative and betalactamase inhibitor with antibacterial activity. Tazobactam contains a beta-lactam ring and irreversibly binds to beta-lactamase at or near its active site. This protects other beta-lactam antibiotics from beta-lactamase catalysis. This drug is used in conjunction with beta-lactamase susceptible penicillins to treat infections caused by beta-lactamase producing organisms. 